\title{
A systematic review of the effects of advance care planning facilitators training programs
}

\author{
Carmen Wing Han Chan, Nancy Hiu Yim Ng ${ }^{*}$, Helen Y. L. Chan, Martin M. H. Wong and K. M. Chow
}

\begin{abstract}
Background: Advance care planning (ACP) is the process of ongoing communication among patients, family and health care professionals regarding what plans for future care are preferred in the event that patients become unable to make their own decisions. Clinicians play an important role in ACP as both initiators and decision coaches. However, lack of training for clinicians has frequently been reported as the reason for low involvement in ACP discussions - hence the present review evaluates the effectiveness of ACP training programs for healthcare professionals to guide the development of novel training programs for them in the future.

Methods: A literature search for intervention studies was conducted independently by two reviewers in July 2018. Participants included all healthcare professionals working with adult patients suffering from terminal illness. The primary outcomes were the professionals' knowledge of and attitudes towards ACP, and self-perceived competence in ACP conversations. The Effective Public Health Practice Project appraisal tool was used to examine the quality of the studies included.
\end{abstract}

Results: A total of 4025 articles were identified, and ten eligible articles, covering 1081 participants, were included in the review. However, there is a lack of high quality randomized controlled trials of providing ACP training for nurses working in non-palliative care hospital settings. The overall quality of the intervention studies was moderate. All the studies included used instructional sessions in their interventions, while some contained group discussion, roleplay and the use of advanced technology. The training programs increased the knowledge, attitudes towards shared decision-making, perceived communication skills, confidence, comfort and experiences concerned with discussing end-of-life (EOL) issues. Patient advocacy, job satisfaction and perceived level of adequate training for EOL care were improved. The use of 'decision aids' was rated as acceptable and clinically useful.

Conclusions: Training for healthcare professionals in ACP has positive effects on their knowledge, attitude and skills. The use of decision aids and advanced technology, instructional sessions with role play, training content focused on ACP communication skills and the needs and experience of patient in the ACP process, and a valuesbased ACP process are all those factors that made the ACP training programs effective.

Keywords: Advance care planning, Palliative care, End-of-life care, Education, Training, Facilitator, Nurse, Healthcare professionals

\footnotetext{
* Correspondence: nhy582@ha.org.hk

The Nethersole School of Nursing, Faculty of Medicine, The Chinese

University of Hong Kong, 6/F, Esther Lee Building, Shatin, NT, Hong Kong
}

(c) The Author(s). 2019 Open Access This article is distributed under the terms of the Creative Commons Attribution 4.0 International License (http://creativecommons.org/licenses/by/4.0/), which permits unrestricted use, distribution, and reproduction in any medium, provided you give appropriate credit to the original author(s) and the source, provide a link to the Creative Commons license, and indicate if changes were made. The Creative Commons Public Domain Dedication waiver (http://creativecommons.org/publicdomain/zero/1.0/) applies to the data made available in this article, unless otherwise stated. 


\section{Background}

Palliative care focuses on the quality of life of patients and their families facing a life-threatening illness, through the prevention and relief of pain and other problems, physical, psychosocial and spiritual [1].

According to the statistics of one regional hospital in Hong Kong, the rate of deaths in acute, non-palliative settings was $91.7 \%$ in 2016, meaning that a significant proportion of deaths occurred in non-palliative care units such as acute medical and surgical units. Healthcare professionals, especially doctors and nurses, working in non-palliative care units and acute care settings would be expected to provide EOL care. Communication and decision-making about the goals of care as identified by seriously ill hospitalized patients and their families are important elements for improvement in the quality of end-of-life (EOL) care [2].

Advance care planning (ACP) is the process of ongoing communication among patient, family and healthcare professional regarding the preferred planning for future care in the event when patients become unable to make their own decisions. Healthcare professionals are expected to play important roles to initiate $\mathrm{ACP}$ and act as decision coaches [3]. ACP provided by trained non-physician facilitators have been shown to increase the convergence of patients' wishes and the EOL care that they receive [3].

An advance directive (AD) is a statement, usually in writing, made by a person to indicate advance refusal of medical treatment and directions on the kind of lifesustaining treatments that should be withheld/withdrawn when he/she is no longer mentally capable of making healthcare decisions. The literature shows that facilitated ACP can increase the number of meaningful and valid ADs, strengthen patient autonomy and improve the quality of care when EOL is near [4-6]. A systematic review by Brinkman-Stoppelenburg, Rietjens and van der Heide [7] found that comprehensive ACP discussion may be more effective than ADs alone in improving compliance with a patient's EOL wishes and satisfaction with care. Findings of the systematic review by Klingler, in der Schmitten and Marckmann [8] even showed that facilitated ACP has the potential to reduce the net costs of care.

However, an integrative review by Rietze and Stajduhar [9] found that nurses in acute care settings had a low involvement in $\mathrm{ACP}$ discussions. Limited education and training in $\mathrm{ACP}$ and EOL care conversations are reported as barriers in facilitating ACP conversations [10]. Lack of education and knowledge, lack of time with patients, communication barriers and symptom management are some of the themes drawn from the literature review by McCourt, Power and Glackin [11] on the provision of EOL care in acute hospital settings.
Lund, Richardson and May [12] suggested that interventions mostly likely to increase the adoption of ACP in clinical practice are those that make elements of ACP workable within complex and time-pressured clinical workflows. The systematic review and meta-analysis by Oczkowski, Chung, Hanvey, Mbuagbaw and You [13] suggested that the use of structured communication tools might increase the communication of preferred care. Another systematic review, by Cardona-Morrell et al. [14], concluded that the available decision aids seemed to enhance patients' and surrogates' knowledge of the care options. Yet the studies included in these systematic reviews were not targeted at ACP training programs for healthcare professionals [12-14]. Chung, Oxzkowski, Hanvey, Mbugbaw, and You [15] conducted a systematic review and meta-analysis on educational interventions and suggested that when compared with usual teaching, healthcare professionals' self-efficacy, knowledge and EOL communication scores might be improved by EOL communication training. However, the finding was only applicable to such training in general, only a very few of the included studies focused on ACP facilitator training [15]. A systematic review was thus conducted to evaluate the effectiveness of ACP training programs for healthcare professionals. The findings will be used to guide the development of a novel program to meet the training needs of healthcare professional and facilitate ACP conversations in acute care settings.

\section{Objectives}

The purpose of this systematic review is to obtain evidence for the effectiveness of an ACP training program for healthcare professionals. The following research questions were to be answered:

(1) What is the effectiveness of the program in improving healthcare professionals' knowledge regarding $\mathrm{ACP}$ ?

(2) What is the effectiveness of the program in improving healthcare professionals' attitude regarding $\mathrm{ACP}$ ?

(3) What is the effectiveness of the program in improving healthcare professionals' competence regarding $\mathrm{ACP}$ ?

(4) What is the effectiveness of the program in increasing the frequency of initiating ACP conversations by healthcare professionals?

\section{Methods}

Eligibility criteria

Types of studies

Randomized controlled trials (RCTs) and quasiexperimental studies with a control group, including 
uncontrolled before-and-after studies in which participants acted as their own control were included. Eligible articles were those written in English in any publication year. Articles written in languages other than English were excluded.

\section{Types of participants}

Participants were various classes of healthcare professionals who were working with adult patients over 18 with terminal illnesses. Population groups in community settings or home-dwelling patients and neonatal/ pediatric patients were excluded.

\section{Types of intervention}

Advance care planning training program for healthcare professionals were included. Training programs targeting patients or family members were excluded.

\section{Types of outcome measures}

The outcomes were healthcare professionals' knowledge of and attitude towards ACP, self-perceived competence in $\mathrm{ACP}$ discussion and the frequency of initiating $\mathrm{ACP}$ conversations.

\section{Search strategies}

A comprehensive search was conducted in July 2018. Initial keywords relevant to the topic were identified by a search of MEDLINE. A more extensive search using the identified keywords was performed using the following eight databases: MEDLINE, CINAHL, PubMed, EMBASE, Cochrane Library, JBI EBP, PsycINFO, Health and Psychosocial Instruments. The keywords were: (Advance Care Planning OR advance medical planning OR advance health care planning OR advance care plan* OR ACP OR Advance Directive* OR decision making OR treatment decision making OR ethical decision making OR living will* OR funeral preparation) AND (palliative care OR palliative therapy OR palliative treatment OR end of life OR hospice OR terminal care OR terminally ill OR death and dying OR life limiting illness* OR life threatening illness*) AND (nurs* OR health person* OR health professional* OR allied health worker* OR facilitator* OR physician* OR social worker* OR surgeon* OR oncologist*) AND (education* OR train* OR workshop OR facilitation) AND (hospital\$ OR in patient* setting OR acute care OR critical care OR intensive care OR rehabilitation OR surgical OR oncolog*). A manual search of the reference list of included articles and relevant journals was undertaken to identify relevant articles for screening.

\section{Study selection}

After the removal of duplicates, two reviewers (NHYN and $\mathrm{MMHW}$ ) independently performed the initial screening on the titles and abstracts according to the eligibility criteria. Full texts were then obtained to determine the eligibility of the whole studies. Discrepancies were solved by group consensus and a final decision by the third reviewer $(\mathrm{CWHC})$.

\section{Data extraction and quality appraisal}

For each of the included studies, two reviewers (NHYN and MMHW) independently extracted the study characteristics: aim, design, population, intervention, measurement tool and results. The methodological quality of all the studies was assessed by means of the Effective Public Health Practice Project (EPHPP) appraisal tool [16], which evaluates an interventional study in eight domains: selection bias, study design, confounders, blinding, data collection methods, withdrawals and dropouts, intervention integrity and analysis. The judgement of the methodological rating was strong, moderate and weak, as evaluated by two reviewers independently (NHYN and MMHW). Whenever a discrepancy occurred, it was solved by group consensus and a final decision by the third reviewer $(\mathrm{CWHC})$.

\section{Results}

\section{Study selection}

A total of 4025 articles were identified. After the screening on the titles and abstracts, A total of 104 articles were potentially eligible and their full texts were retrieved and reviewed. Finally, ten articles were included in the review [17-26]. A PRISMA flow chart of the study retrieval and selection process with reasons for exclusion at each stage is provided in Fig. 1.

\section{Participants and settings}

The details of the ten studies are given in Table 1. A total of 1081 participants were included in the review. The sample in each study varied from 16 to 278 , with five $[19,20,22,24,25]$ including more than 100 participants. Five studies were conducted in the US [17, 20, 22, 25, 26], two in Australia [19, 24], one in the UK [18], one in Canada [23] and one in Korea [21]. The majority of participants were nurses, physicians and medical students. The participants in three studies were recruited from hospitals $[19,23,24]$, in three from internal medicine units $[17,18,25]$ including renal medicine [18], in two from critical care units $[21,26]$ and in another two from students undergoing the usual medical curriculum $[20,22]$.

\section{Study interventions}

All included studies involved instructional sessions in their interventions [17-26], while the interventions in seven studies contained discussions among participants $[17-20,22,23,25]$, and four included role-play [17-19, 


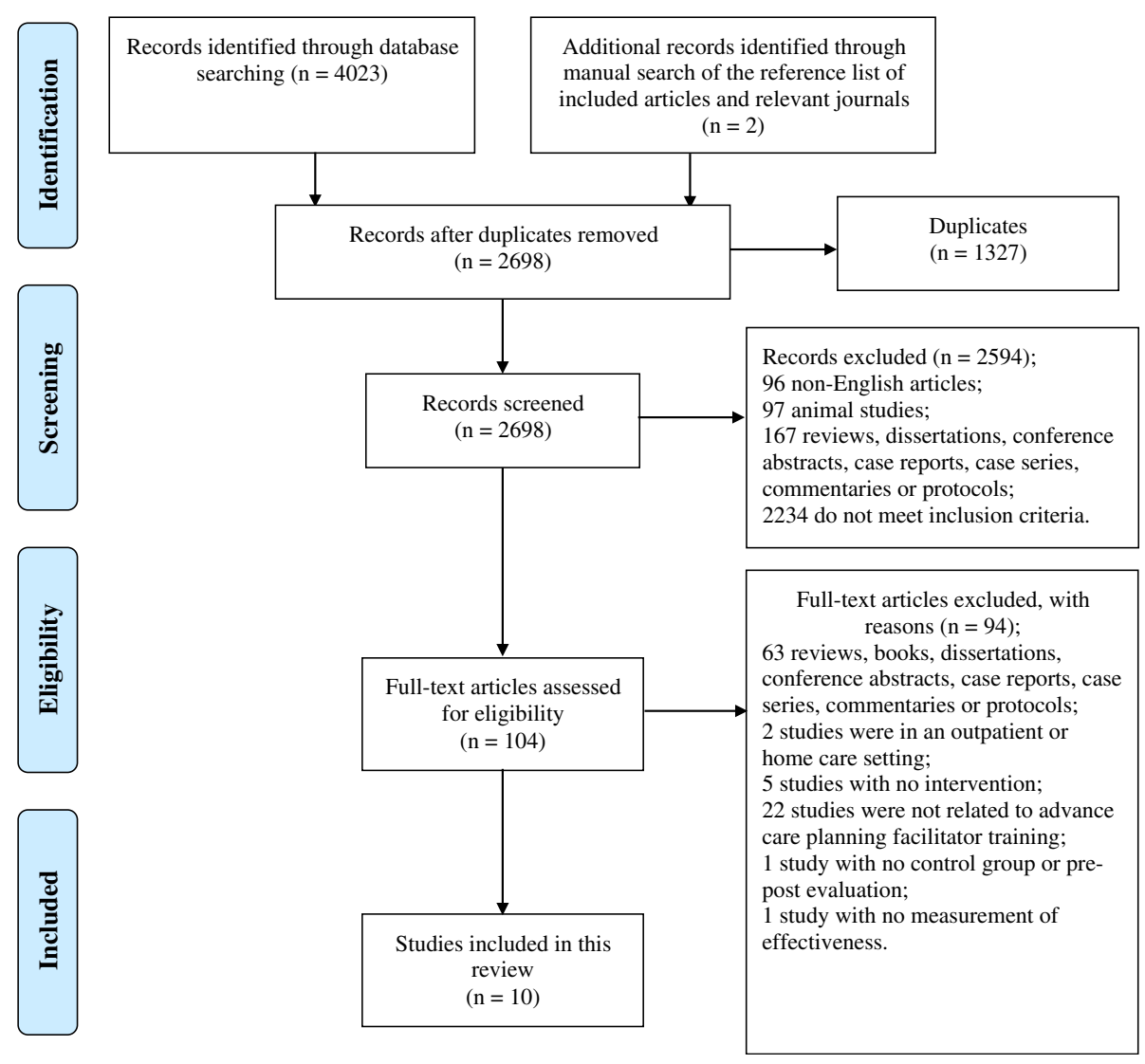

Fig. 1 PRISMA flowchart of the study retrieval and selection process

25]. Advanced technology, such as audio-visual materials [17, 19, 20, 22], online tutorials [23] and interactive patient e-simulation [19], were also used. Regarding the training content, the majority focused on ACP communication skills $[17,21,25,26]$ and the needs and experience of patient in the ACP process [17, 18, 22]. Intensity of the intervention varied from 75-min values-based ACP educational session (Lum et al. [22]) to 2-3 h seminar /workshop (Greenberg et al. [20]), Detering et al. [19]), $8 \mathrm{~h}$ (Jo and An [21], Smith [25]), 2 days training (Alexander et al. [17], Bristowe et al. [18], Seal [24]) over a duration of 4 to 8 weeks (Jo and An [21], Murray et al. [23], Wilson et al. [26]). Of the 10 studies, only two studies (Alexander et al. [17] and Murray et al. [23]) scored high quality using EPHPP. As for program structure, Alexander et al. [17] used a short course (two-day retreat/ 16-h curriculum) to improve physicians' communication skills in delivering bad news and eliciting patients' preferences for EOL care. It has three components: control of pain and symptom management; communication skills; and sessions designed to promote participants' understanding of patients and families' experience, enhance their personal awareness, and inform them about ethical issues. Murray et al. [23] also used three components in the intervention - online tutorial 10 modules with quizzes and feedback; skill-building workshop with use of performance feedback, video exemplar, place of care patient decision aid, case studies, and practice and feedback; and educational outreach. The educational program of Jo and An [21] included a series of eight sessions of $60 \mathrm{~min}$, twice a week for 4 weeks. Each session was designed with a specific topic related to shared decision-making and determined by an expert panel [21]. The study by Seal [24] was mainly targeted at nurses and the intervention group received the Respecting Patient Choices Program (RPCP), which was part of a national palliative care program. Lum et al. [22] applied 'Conversation Starter Kits', a free downloadable handout emphasizing a values-based rather than a procedure-based ACP process. Bristowe et al. [18] conducted a survey on end-stage kidney disease patients in order to identify the specific needs of those participants cared for before the program. The educational intervention by Smith et al. [25] consisted of two one-hour 'lunch conferences' involving role-play, and six one-hour morning sessions discussing real-time cases. The participants in the high-intervention group of Greenberg et al. [20] were required to initiate AD discussion with a 


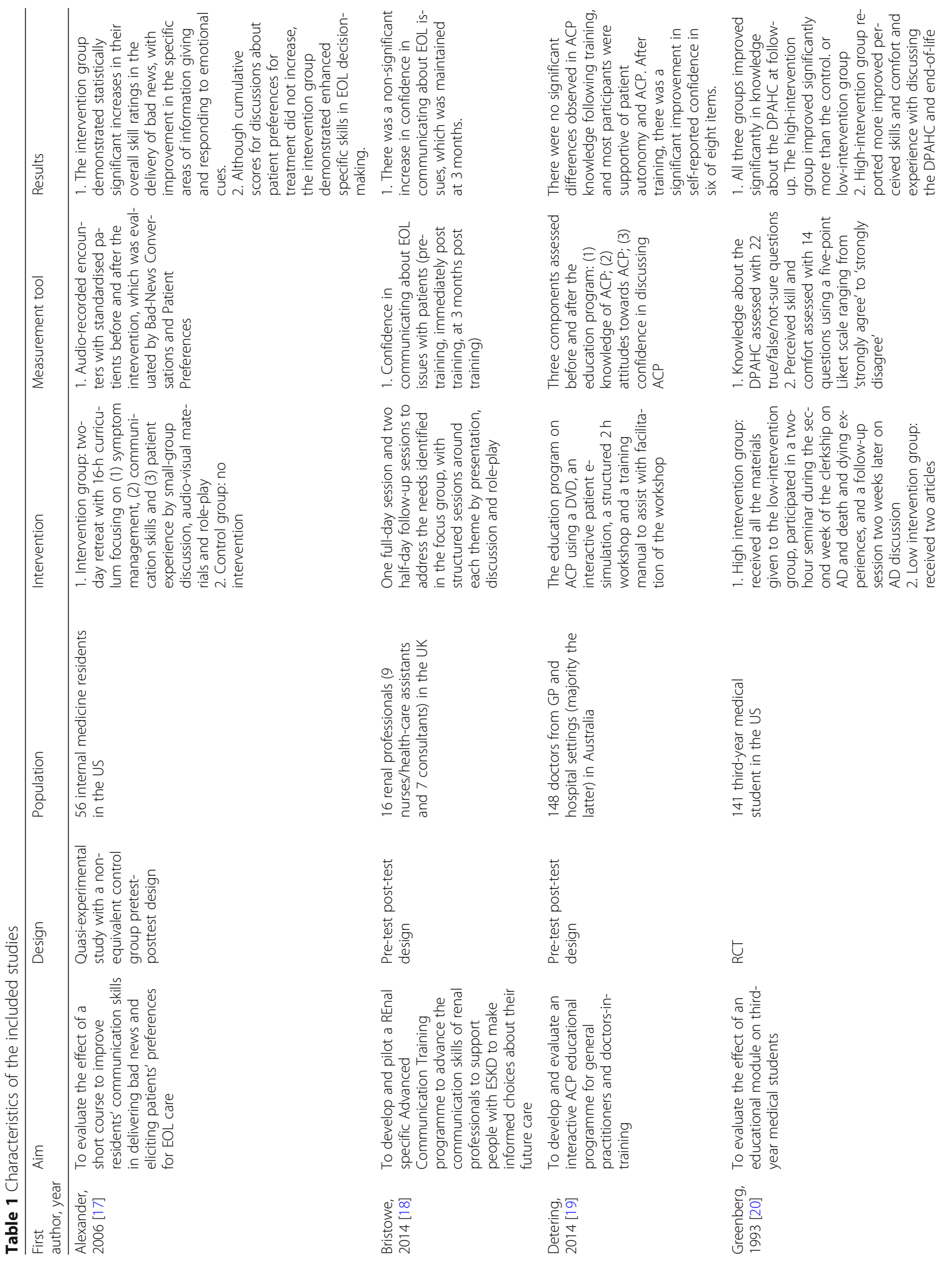




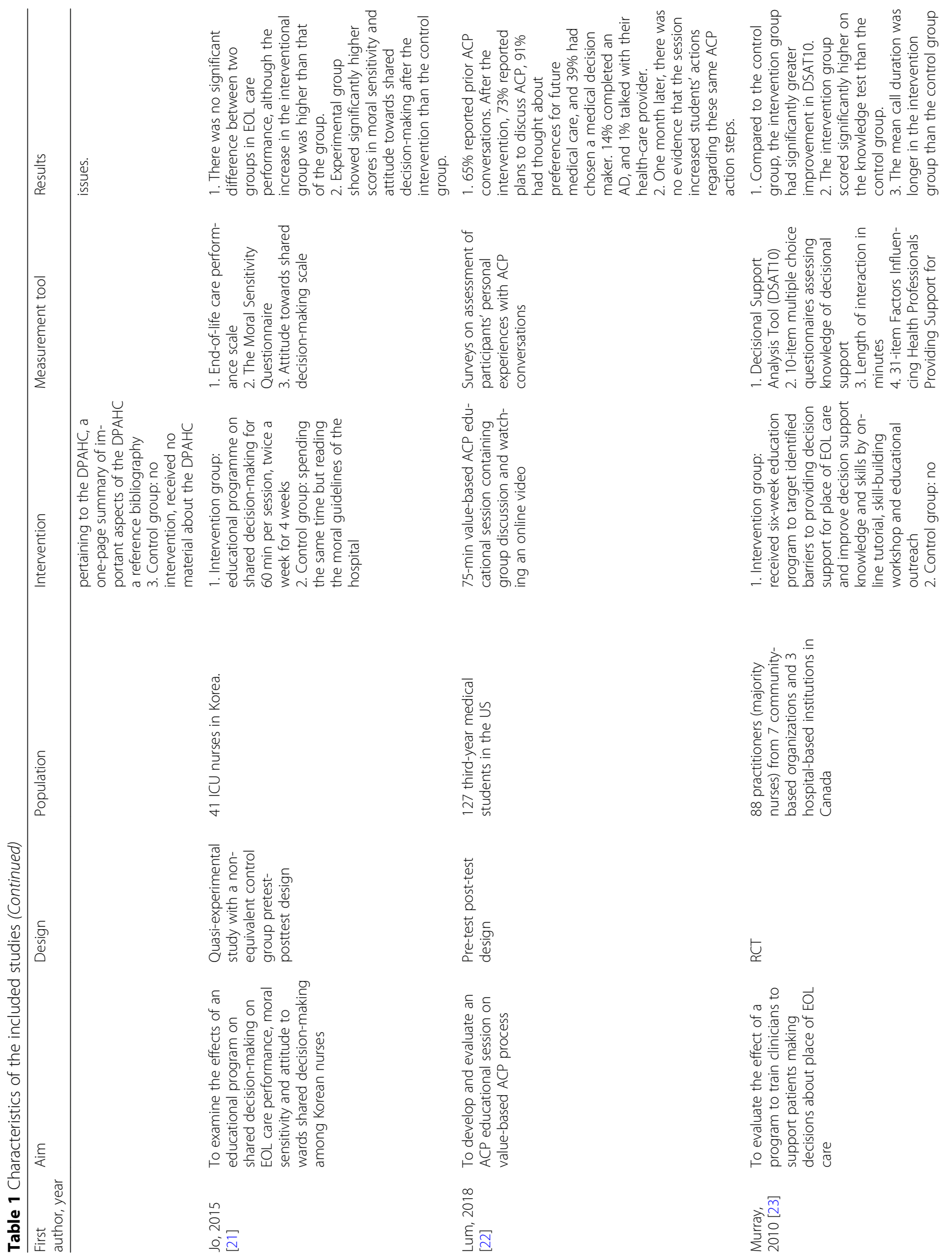




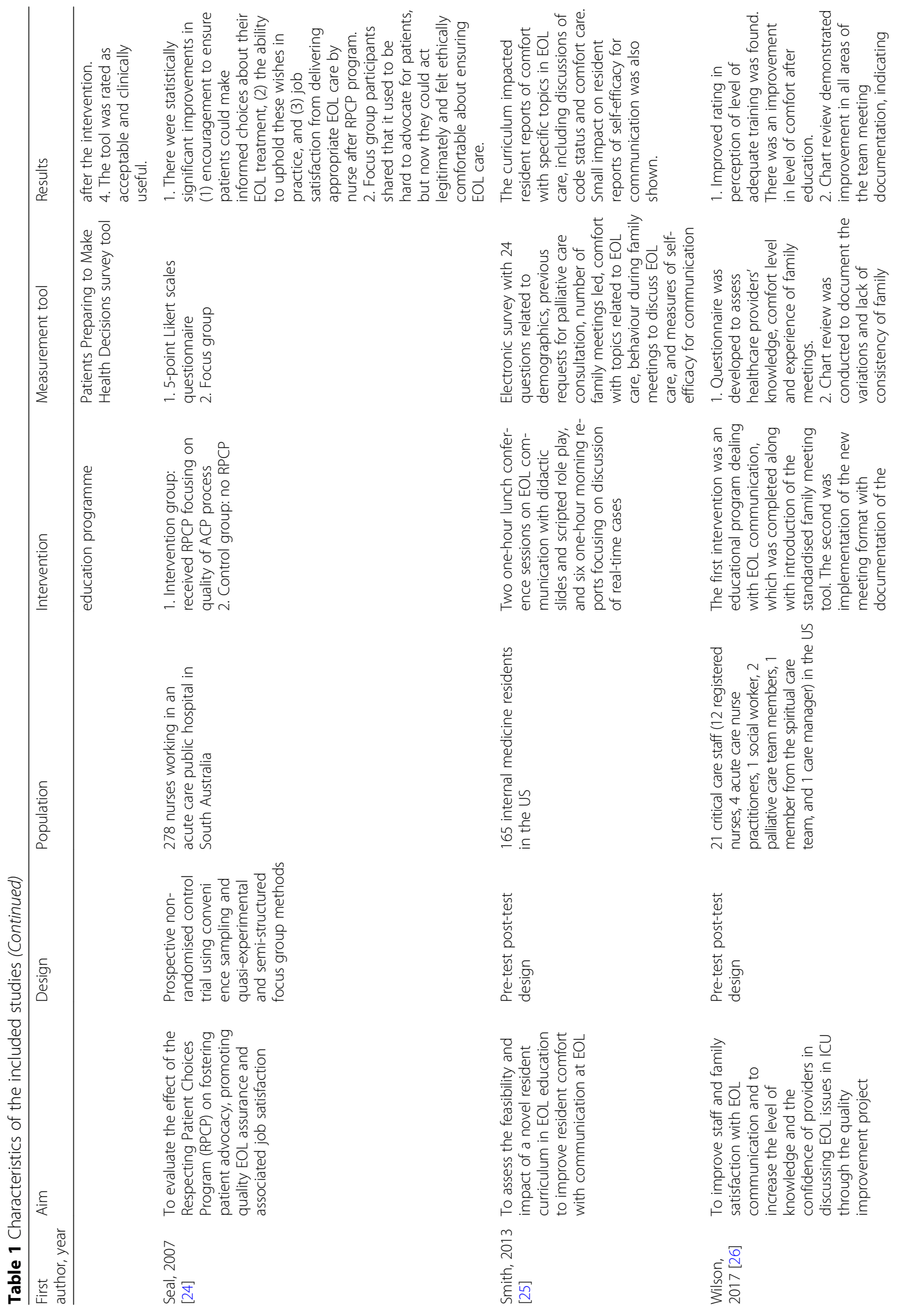




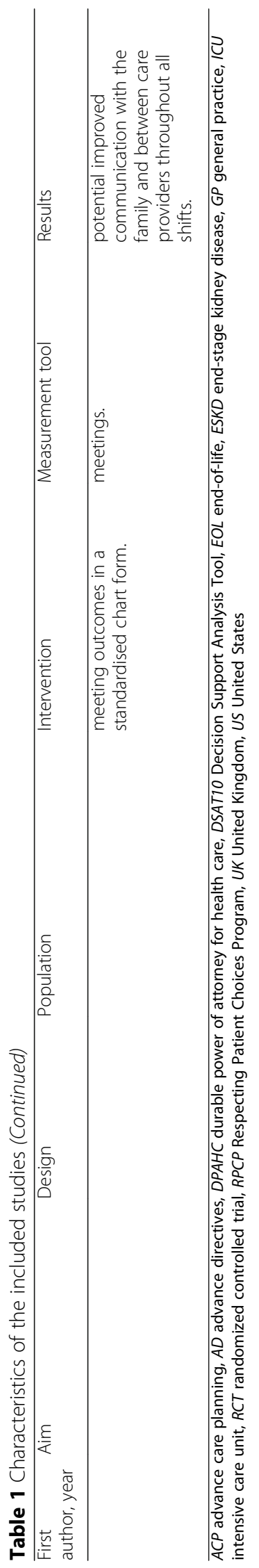




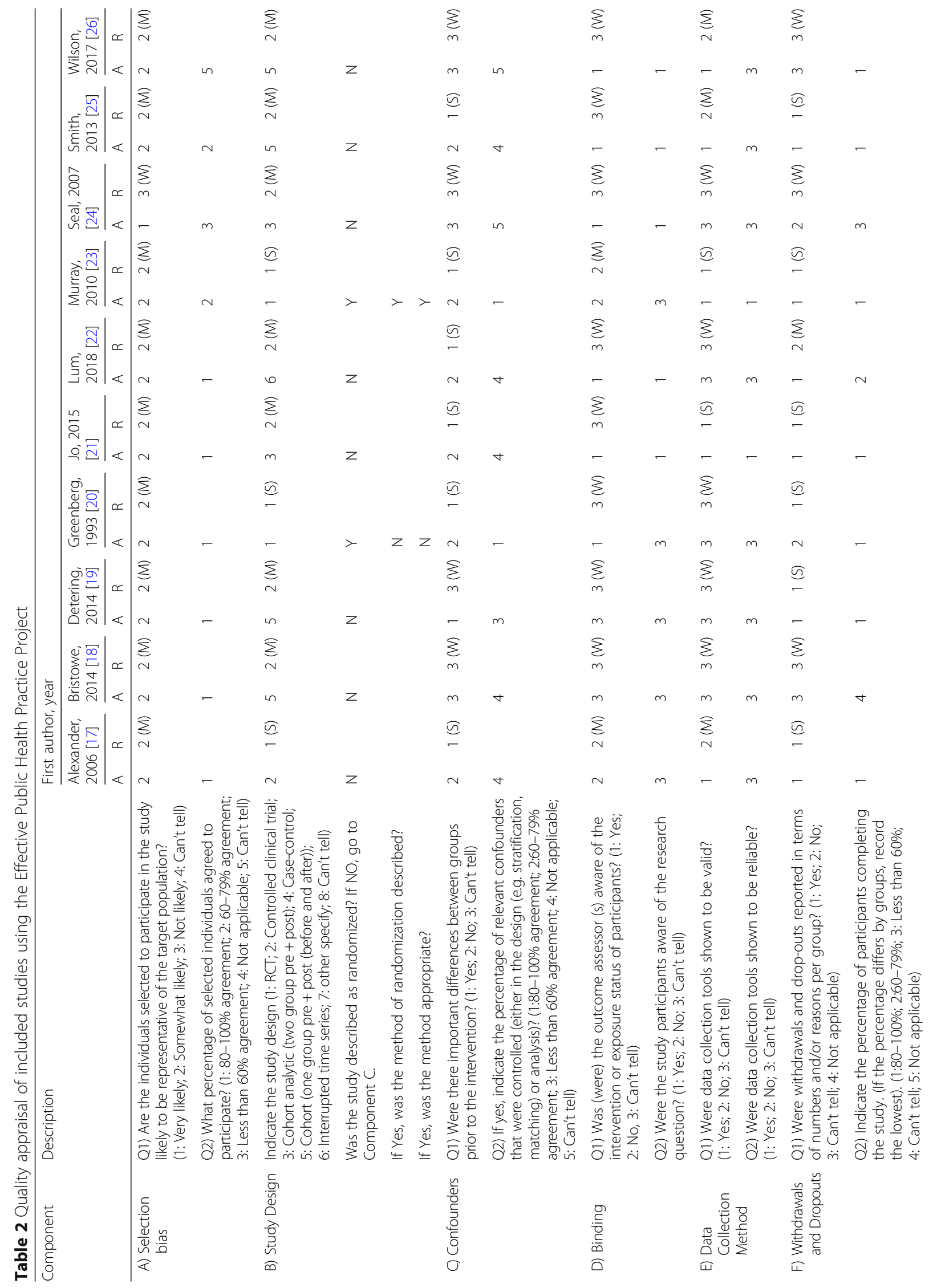




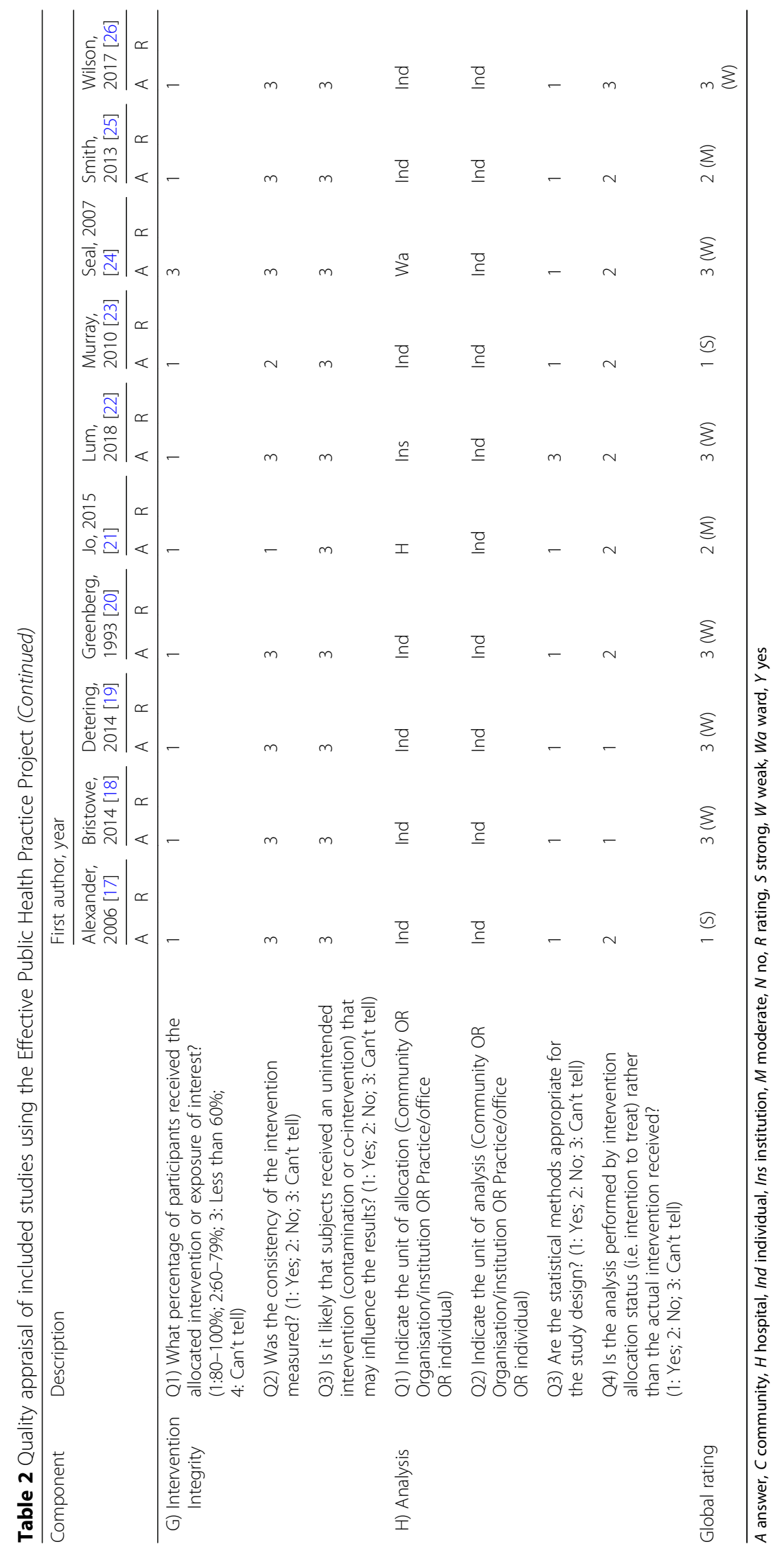


patient, family member or friend to apply what they had learnt in the earlier seminar. To train healthcare professionals facilitating the ACP process, a novel feature of the intervention of Murray et al. [23] was that it involved a specific patient decision aid (PtDA) for practitioners to guide patients in place of EOL care discussions, although the PtDA only focused on the place of care decision making instead of a broader coverage of all relevant EOL healthcare decision-making in the ACP process. Wilson et al. [26] involved the use of standardized family meeting tool with documentation of the meeting outcomes in the intervention to improve EOL communication.

\section{Outcome measures}

The outcome measures of the studies were mainly trainee-related outcomes, including knowledge, competence and skills in ACP after the educational program. In the study by Murray et al. [23], the primary outcome measure was the change in the quality of decision support provided by practitioners to a standardized patient before and after the intervention, while Alexander et al. [17] also used standardized patients to evaluate residents' communication skills. The use of a standardized patient to collect evaluation data is more objective than a self-assessed performance, as self-reported changes are unverifiable. Secondary outcome measures used by Murray et al. [23] were knowledge, duration of interaction, intention to engage in patient decision support and acceptability of intervention components, including the decision tool, which were comprehensive and reflected the effectiveness of the training program from different aspects. A substantial number of studies adapted validated tools, e.g. Decision Support Analysis, Factors Influencing Health Professionals Providing Support for Patients Preparing to Make Health Decisions, Bed-News Conversations and Patient Preferences, to measure the outcomes. Jo and An [21] used the end-of-life care performance scale developed by Park [27], the Moral Sensitivity Questionnaire developed by Lutzen et al. [28], and attitudes towards shared decision-making scale developed by Jo as their outcome measures. A semistructured focus group was used by Seal [24] to give qualitative data on participants' perceptions of the program. The least productive of evidence was the use of non-validated questionnaires/surveys as in the studies of Bristowe et al. [18], Detering et al. [19], Greenberg et al. [20], Seal [24] and Lum et al. [22, 25]. Except Alexander et al. [17], Smith et al. [25], and Wilson et al. [26], all mentioned about the time course of their follow up measurements from immediately post training to 2 weeks (Detering et al. [19], Murray et al. [23]), 4 weeks (Jo and An [21], Lum et al. [22]), 6 weeks (Greenberg et al. [20]), 3 months (Bristowe et al. [18]), and 6 months (Seal [24]) after training. All except Lum et al. [22] reported intervention effect maintained at time of follow up. Lum et al. [22] reported no evidence that the training session increased participants' actions regarding ACP action steps one-month post training. They commented that the one-month follow up period may not have provided adequate time for the participants to take $\mathrm{ACP}$ action, which is a limitation of such study design.

\section{Effects of interventions}

All the included intervention studies showed positive results. The training programs significantly increased knowledge [20, 23], attitudes towards shared decisionmaking [21], perceived communication skills [17, 20, $23]$, confidence $[19,25]$, comfort $[20,25,26]$ and experiences [20] in discussing EOL issues. Bristowe et al. [18] and Jo and An [21] reported non-significant increases in confidence in EOL communication and performance respectively. Wilson et al. [26] found improvement in all areas of team-meeting communication after intervention. The mean duration of interaction with standardized patients was longer in the intervention than the control group after the intervention by Murray et al. [23]. Lum et al. [22] found that $90 \%$ of participants evaluated the educational value of the session positively. However, there was no evidence that the session increased students' actions regarding the ACP steps after 1 month. Lum et al. [22] explained that the one-month follow-up period might not provide the students with adequate time to take ACP action. The study by Seal [24] fostered patient advocacy by nurses and associated job satisfaction, as nurses thought that they could deliver appropriate EOL care to patients after the program. Improved rating of perceived levels of adequate training for EOL care was found by Wilson et al. [25, 26]. The study by Murray et al. [23] revealed that participants welcomed the use of decision tools in ACP communication, as they were rated as acceptable and clinically useful.

\section{Methodological quality}

Detailed information on the methodological quality of each included study is presented in Table 2. Quality assessment of the ten studies using EPHPP showed two to be strong $[17,23]$, two moderate $[21,25]$ and six weak $[18-20,22,24,26]$ in the global rating. Concerning selection bias, one [24] was weak as less than $60 \%$ of the selected individuals agreed to participate. Of the other nine studies, six [17-22] had $80-100 \%$ agreement. As for study design, two were RCTs [20, 23], two were quasi-experimental with a non-equivalent control group pretest-posttest design $[17,21]$, one was a prospective non-randomized control trial using convenience sampling and quasi-experimental and semi-structured focus group methods [24], and the others were cohort (one 
group pre \& post) $[18,19,22,25,26]$. Except the study conducted by Detering et al. [19], there were no important differences between groups prior to the intervention, or no control group was presented in all other studies. The outcome assessors were not blinded to the intervention or exposure status of participants in the studies of Greenberg et al. [20], Jo and An [21], Lum et al. [22], Seal [24], Smith et al. [25] and Wilson et al. [26]. Except for Bristowe et al. [18], Detering et al. [19], Greenberg et al. [20], Seal [24] and Lum et al. [22], where the validity of the data collection tools was not mentioned, all the other studies showed valid data collection tools. All except Seal [24] reported attrition numbers, and in that study the percentage of participants completing the program was less than $60 \%$.

\section{Discussion}

\section{Implications for clinical practice}

The studies included in this review showed that ACP education or training for healthcare professionals has positive effect on the knowledge, attitude, skills and comfort of participants in discussing issues related to EOL decision-making. With adequate training and skills transfer, doctors and nurses in non-palliative care settings can be equipped with the appropriate attitudes, knowledge and skills for conducting ACP, to address patients' and their families'/carers' needs and preferences regarding their care. However, not many studies measured the effect of ACP facilitator training programs on frequency of initiating ACP discussions and there was a lack of strong evidence for an effect of ACP facilitator training programs on frequency of initiating ACP discussions. Lund et al. [12] underscored the impact of clinical and organizational pressures on implementation of ACP. Multiple and competing demands of other work, problems in sharing decisions and preferences of patients within and between healthcare organizations, and the availability and preparation of staff are the barriers highlighted that affect opportunities and frequency to initiate and operationalize ACP discussions in clinical settings. Barnes [29], Ke et al. [30], Johnson, Butow, Kerridge and Tattersall [31] also pointed out that clinical doctors and nurses in acute settings have a heavy workload and insufficient time to conduct ACP, especially when they have other clinical tasks to undertake; and ACP is also influenced by the organizational culture. Future study should explore ways to tackle the barriers and more studies should measure the effect of ACP facilitator training programs on frequency of initiating $\mathrm{ACP}$ discussions. Appropriate use of decision aids may be explored, to be used in clinical settings to assist the ACP discussion. Simple decision-making tools may likely increase the likelihood of their adoption and normalization in practice as well as increase patients' willingness to engage with them [12]. Organizational support is a key success factor in implementing an ACP facilitator training program for healthcare professionals working in non-palliative care hospital settings. Organizational support entails the organizational commitment to identifying, documenting, sharing and acting upon patients' preferences [12], promulgation of ACP facilitation in all settings, encouraging the incorporation of ACP discussions into the clinical practice of frontline clinicians through clinical governance procedures, and providing training to clinicians on ACP facilitation. It is foreseen that extensive discussion with hospital administrators and managers will be necessary for an additional workforce to implement ACP and to release frontline clinicians to receive the training program. Regular in-service training and workshops on ACP should also be arranged for newly joined clinical staff to ensure the sustainability of ACP discussion as a normal practice in non-palliative acute care settings.

\section{Implications for future research}

The results of this review also showed that there were limited high quality RCTs conducted internationally to evaluate the effectiveness of ACP facilitator training programs for healthcare professionals working in nonpalliative care hospital settings. Although one of the included studies used decision tools to train practitioners facilitating ACP discussion [23], the systematic review by Cardona-Morrell et al. [14] implied that decision aids for initiating or terminating EOL treatment were scarce and lacked prognostic information on the pros and cons of alternative treatment options and preferences. More systematic evaluation of the effectiveness of a comprehensive decision aid is needed, aid that includes patient values (such as quality EOL, physical functioning, burden on families and attitudes to risk) and other important information like prognosis, treatment goals, and pros and cons of treatment, to guide patients and their surrogates to make EOL decisions, so that decision aids may be included to facilitate communication and understanding of the patient experience in future ACP facilitator training programs.

\section{Recommendations for the intervention to be developed}

In summary, there is limited interventional study on training healthcare professionals from hospital settings as ACP facilitators, but there is a great demand for it [3, 22]. Training programs on ACP discussions should be considered that include teaching sessions containing small group discussion and communication skills training/workshops providing opportunities to practise the specific skills through role play with the use of appropriate decision aids [14, 17, 23]. Advanced technology such as interactive patient e-simulation can be used to 
provide more practice for trainees [19]. Values-based instead of procedure-based ACP educational sessions should be adopted. The content of the Conversation Starter Kit could be used as the frame for values-based ACP discussion [22], as it is a free downloadable handout, which makes it more accessible. The Respecting $\mathrm{Pa}$ tient Choices Program (RPCP) can be considered as a model for training nurses [24]. The systematic review by Myers et al. [32] found that Respecting Choices or tools based on that program were the ACP provider tools most often encountered in the literature, and suggested it was a critical strategy for affecting patient outcomes. Furthermore, similar to the study by Bristowe et al. [18], patient surveys can be conducted to identify the needs of patients that the target participants serve, and discussion with patient, family member or friend can be fostered to apply what the participants have learnt from the training program [20].

As for outcome measurement, qualitative data on patients and families' perceptions of and satisfaction with ACP implementation by healthcare professionals by structured interview can be considered.

The relatively short, intensive course in Alexander et al. [17] yielded statistically significant positive results, and so may be taken as a reference for the intervention design of the future training program. However, Alexander et al. [17] designed the course mainly for US medical residents. The eight training sessions developed by Jo and An [21] for Korean nurses may also be considered as a reference. A qualitative interview is needed to understand healthcare professionals' perceptions of ACP implementation by clinicians working in non-palliative care hospital settings to inform the development of the training program.

\section{Strengths and limitations}

This is the first systematic review evaluating the effects of ACP facilitator training programs for healthcare professionals. The methodology adhered to the PRISMA statement [33] and the quality of each study was critically assessed using EPHPP [16].

Although each study contained clear descriptions of objectives, the intervention, outcome measurement and study finding, meta-analysis cannot be performed because the intervention and outcome measurements are so varied. However, positive results from all the studies indicated that ACP facilitator training programs for healthcare professionals were effective in increasing their knowledge, attitudes and skills in ACP.

\section{Conclusions}

Communication and decision-making about the goals of care are identified by seriously ill hospitalized patients and their families as important targets for improvement, if the quality of EOL care is to be enhanced [2]. Although a significant proportion of deaths occur in nonpalliative care settings, clinicians in acute settings have low involvement in ACP discussions [9]. The importance of ACP in clarifying patients' values and respecting their wishes or autonomy is clear. Nurses have a valuable role in leading ACP implementation and in creating systemwide cultural changes to improve EOL care [34]. This systematic review found that training for healthcare professionals in ACP had positive effects on their knowledge, attitude and skills. However, there is a lack of high quality $\mathrm{RCTs}$ to evaluate the effectiveness of ACP facilitator training programs for nurses working in non-palliative care hospital settings. By evaluating the effectiveness of the training programs, the possibilities of such programs in clinical practice will be explored and recommendations for further development of ACP training program will be made, to enhance quality EOL care in non-palliative care hospital settings. In conclusion, the use of decision aids and advanced technology, instructional sessions with role play, training content focused on ACP communication skills and the needs and experience of patient in the $\mathrm{ACP}$ process, and a values-based ACP process are all those factors that made the ACP training programs effective.

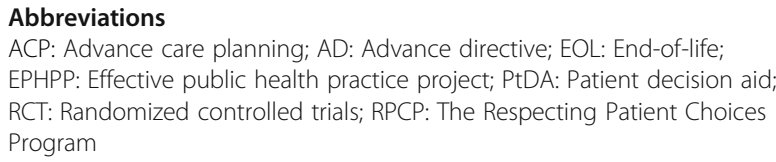

Acknowledgements

Not applicable.

\section{Authors' contributions}

All authors have made substantial contributions to this review and given the final approval of the version to be submitted. NHYN designed the search strategy, drafted and revised the manuscript. CWHC, HYLC, MMHW and KMC modified the search strategy and research questions, designed and critically revised the draft for important intellectual content. MMHW involved in study retrieval and selection part and critically revised the quality assessment of the studies.

Funding

This study did not receive any grant from funding agencies.

Availability of data and materials

All data generated or analyzed during this study are included in this published article.

Ethics approval and consent to participate

Not applicable. This is a systematic review.

Consent for publication

Not applicable. This is a systematic review.

Competing interests

The authors declare that they have no competing interests. 
Received: 4 September 2018 Accepted: 28 May 2019

Published online: 07 June 2019

\section{References}

1. World Health Organization. WHO definition of palliative care. 2018. http:// www.who.int/cancer/palliative/definition/en/. Accessed 3 Aug 2018.

2. Heyland DK, Cook DJ, Rocker GM, Dodek PM, Kutsogiannis DJ, Skrobik Y, et al. Defining priorities for improving end-of-life care in Canada. CMAJ. 2010; 182(16):E747-E52.

3. You JJ, Downar J, Fowler RA, Lamontagne F, Ma IWY, Jayaraman D, et al. Barriers to goals of care discussions with seriously ill hospitalized patients and their families a multicenter survey of clinicians. JAMA Intern Med. 2015; 175(4):549-56

4. in der Schmitten J, Lex K, Mellert C, Rothärmel S, Wegscheider K, Marckmann G. Implementing an advance care planning program in German nursing homes: results of an inter-regionally controlled intervention trial. Dtsch Arztebl Int. 2014;111(4):50-7.

5. Hammes BJ, Rooney BL, Gundrum JD. A comparative, retrospective, observational study of the prevalence, availability, and specificity of advance care plans in a county that implemented an advance care planning microsystem. J Am Geriatr Soc. 2010;58(7):1249-55

6. Detering $\mathrm{KM}$, Hancock $A D$, Reade $M C$, Silvester $W$. The impact of advance care planning on end of life care in elderly patients: randomised controlled trial. BMJ. 2010:340:c1345.

7. Brinkman-Stoppelenburg A, Rietjens JA, van der Heide A. The effects of advance care planning on end-of-life care: a systematic review. Palliat Med. 2014;28(8):1000-25.

8. Klingler $\mathrm{C}$, in der Schmitten J, Marckmann G. Does facilitated advance care planning reduce the costs of care near the end of life? Systematic review and ethical considerations. Palliat Med. 2016;30(5):423-33.

9. Rietze L, Stajduhar K. Registered nurses' involvement in advance care planning: an integrative review. Int J Palliat Nurs. 2015;21(10):495-503.

10. Duke G, Thompson S. Knowledge, attitudes and practices of nursing personnel regarding advance directives. Int J Palliat Nurs. 2007;13(3):109-15.

11. McCourt R, Power JJ, Glackin M. General nurses' experiences of end-of-life care in the acute hospital setting: a literature review. Int J Palliat Nurs. 2013; 19(10):510-6.

12. Lund S, Richardson A, May C. Barriers to advance care planning at the end of life: an explanatory systematic review of implementation studies. PLoS One. 2015;10(2):e0116629.

13. Oczkowski SJ, Chung H-O, Hanvey L, Mbuagbaw L, You JJ. Communication tools for end-of-life decision-making in ambulatory care settings: a systematic review and meta-analysis. PLoS One. 2016;11(4):e0150671.

14. Cardona-Morrell M, Benfatti-Olivato G, Jansen J, Turner RM, FajardoPulido D, Hillman K. A systematic review of effectiveness of decision aids to assist older patients at the end of life. Patient Educ Couns. 2017;100(3):425-35.

15. Chung H-O, Oczkowski SJ, Hanvey L, Mbuagbaw L, You JJ. Educational interventions to train healthcare professionals in end-of-life communication: a systematic review and meta-analysis. BMC Med Educ. 2016;16(1):131.

16. Thomas BH, Ciliska D, Dobbins M, Micucci S. A process for systematically reviewing the literature: providing the research evidence for public health nursing interventions. Worldviews Evid-Based Nurs. 2004;1(3):176-84.

17. Alexander SC, Keitz SA, Sloane R, Tulsky JA. A controlled trial of a short course to improve residents' communication with patients at the end of life. Acad Med. 2006:81(11):1008-12.

18. Bristowe K, Shepherd K, Bryan L, Brown H, Carey I, Matthews B, et al. The development and piloting of the REnal specific advanced communication training (REACT) programme to improve advance care planning for renal patients. Palliat Med. 2014:28(4):360-6.

19. Detering K, Silvester W, Corke C, Milnes S, Fullam R, Lewis V, et al. Teaching general practitioners and doctors-in-training to discuss advance care planning: evaluation of a brief multimodality education programme. BMJ Support Palliat Care. 2014;4(3):313-21.

20. Greenberg JM, Doblin BH, Shapiro DW, Linn LS, Wenger NS. Effect of an educational program on medical students' conversations with patients about advance directives: a randomized trial. J Gen Intern Med. 1993;8(12): 683-5.

21. Jo KH, An GJ. Effects of an educational programme on shared decisionmaking among Korean nurses. Int J Nurs Pract. 2015;21(6):839-46.
22. Lum HD, Dukes J, Church S, Abbott J, Youngwerth JM. Teaching medical students about "the conversation": an interactive value-based advance care planning session. Am J Hosp Palliat Care. 2018;35(2):324-9.

23. Murray MA, Stacey D, Wilson KG, O'Connor AM. Skills training to support patients considering. J Palliat Care. 2010;26(2):112-21.

24. Seal M. Patient advocacy and advance care planning in the acute hospital setting. Aust J Adv Nurs. 2007;24(4):29-36.

25. Smith L, O'Sullivan $\mathrm{P}, \mathrm{LO} \mathrm{B}, \mathrm{Chen} \mathrm{H}$. An educational intervention to improve resident comfort with communication at the end of life. J Palliat Med. 2013; 16(1):54-9.

26. Wilson T, Haut C, Akintade B. Improving health care provider communication in end-of-life decision-making. AACN Adv Crit Care. 2017; 28(2):124-32.

27. Park S. A study on the degree of death orientation and terminal care performance of nurses. Gwangju: Chonnam National University; 1996.

28. Lutzen K, Evertzon M, Nordin C. Moral sensitivity in psychiatric practice. Nurs Ethics. 1997:4(6):472-82.

29. Barnes L. Advance care planning: considerations for clinical practice. Cancer Nurs Pract. 2017;16(2):32-9.

30. Ke LS, Huang $X, O^{\prime}$ Connor M, Lee $S$. Nurses' views regarding implementing advance care planning for older people: a systematic review and synthesis of qualitative studies. J Clin Nurs. 2015:24(15-16):2057-73.

31. Johnson S, Butow P, Kerridge I, Tattersall M. Advance care planning for cancer patients: a systematic review of perceptions and experiences of patients, families, and healthcare providers. Psychooncology. 2016;25(4):362-86.

32. Myers J, Cosby R, Gzik D, Harle I, Harrold D, Incardona N, et al. Provider tools for advance care planning and goals of care discussions: a systematic review. Am J Hosp Palliat Care. 2018;35(8):1123-32.

33. Moher D, Liberati A, Tetzlaff J, Altman DG. Preferred reporting items for systematic reviews and meta-analyses: the PRISMA statement. Ann Intern Med. 2009:151(4):264-9.

34. Izumi S. Advance care planning: the nurse's role. Am J Nurs. 2017;117(6):56-61.

\section{Publisher's Note}

Springer Nature remains neutral with regard to jurisdictional claims in published maps and institutional affiliations.

Ready to submit your research? Choose BMC and benefit from:

- fast, convenient online submission

- thorough peer review by experienced researchers in your field

- rapid publication on acceptance

- support for research data, including large and complex data types

- gold Open Access which fosters wider collaboration and increased citations

- maximum visibility for your research: over $100 \mathrm{M}$ website views per year

At BMC, research is always in progress.

Learn more biomedcentral.com/submissions 